\title{
AVALIAÇÃO DE GENÓTIPOS DE BANANEIRA À Colletotrichum musae EM PÓS-COLHEITA ${ }^{1}$
}

\author{
DANILO BATISTA PINHO르. EDSON HIYDU MIZOBUTSI ${ }^{3}$, \\ SEBASTIÃO DE OLIVEIRA E SILVA ${ }^{4}$, SIDNEY TAVARES DOS REIS 5 , \\ GISELE POLETE MIZOBUTSI ${ }^{6}$, ADELICA APARECIDA XAVIER $^{7}$, \\ REGINA CÁSSIA FERREIRARIBEIRO ${ }^{8}$, VICTOR MARTINS MAIA ${ }^{9}$
}

RESUMO - Alguns hibridos tetraploides apresentam resistência à antracnose, porém são reduzidas as informações sobre os níveis de resistência desses genótipos e o comportamento dos mesmos no Brasil. O objetivo do presente trabalho foi avaliar a incidência e a severidade de antracnose sobre o comportamento de diferentes genótipos de bananeiras resistentes a Sigatoka-Negra em função de diferentes concentrações de conídios de Colletotrichum musae em diferentes épocas de avaliação. Os frutos colhidos no estádio de maturação fisiológica dos genótipos, Thap Maeo, Caipira, Pacovan Ken, Ambrosia, PV 42-53, PA 42-44, FHIA 01, FHIA 18 e a cultivar Prata-Anã utilizada como testemunha. Buquês contendo três frutos foram atomizados com diferentes concentrações $\left(0 ; 10^{2} ; 10^{3} ; 10^{4} ; 10^{5}\right.$ e $10^{6}$ conídios $\left./ \mathrm{mL}\right)$ de $C$. musae. Os frutos foram incubados a $25^{\circ} \mathrm{C}$. A incidência e severidade da antracnose foi avaliada aos $3 ; 6 ; 9$ e 12 dias após a inoculação. Foi utilizado o delineamento inteiramente casualizado, em esquema fatorial 9x6x4 (genótipos, concentrações de esporos e épocas de avaliação), com quatro repetições, contendo quatro buquês de três frutos. Aos doze dias de avaliação, todos os frutos de todos os genótipos estudados apresentaram incidência de C. musae quando inoculados com as diferentes concentrações de inóculo. A cultivar Thap Maeo apresentou a menor severidade $(27,5 \%)$ de antracnose, enquanto os genótipos Ambrosia, PV 42-53, FHIA 18 e FHIA 01 foram as mais suscetíveis à antracnose com $64 \%, 64 \%, 61,33 \%$ e $58 \%$ de severidade, respectivamente. Termos para indexação: Musa, Cultivares, Antracnose, Doenças, Pós-colheita.

\section{EVALUATION OF GENOTYPES OF BANANA TO Colletotrichum musa IN POSTHARVEST}

\begin{abstract}
Some tetraploides hybrids present resistance to anthracnose, however the information on the levels of resistance of these genotypes and the behavior in Brazil are reduced. The aim of this study was to evaluate the incidence and severity of injury on the behavior of different banana genotypes resistant to Black Sigatoka for different concentrations of conidia of Colletotrichum musae in different times of evaluation. The fruits of the genotypes, Thap Maeo, Caipira, Pacovan Ken, Ambrosia, PV 42-53, PA 42-44, FHIA 01, FHIA 18 and cultivar Prata Anã, were collected at the stage of physiological maturation. Bunches containing three fruits were atomized with different concentrations: $0,10^{2}, 10^{3}, 10^{4}, 10^{5}$ and $10^{6}$ of conidia/mL of C. musae. The fruits were incubated at $25^{\circ} \mathrm{C}$. The incidence and severity of the anthracnose were evaluated $3,6,9$ and 12 days after the inoculation. A completely randomized experimental design was used, in a factorial scheme 9x6x4 (genotypes, concentrations of spores and evaluation periods) with four replications containing four bunches of three fruits. After twelve days of evaluation all the fruits of all the studied genotypes presented incidence of $C$. musae when different concentrations of inoculum were used. Concerning the severity of the anthracnose, the cultivar Thap Maeo had the lowest severity $(27,5 \%)$ of anthracnose while the genotypes Ambrosia, PV 42-53, FHIA 18 and FHIA 01 were the most susceptible to the anthracnose at $64 \%, 64 \%$, $61,33 \%$, and $58 \%$ of severity, respectively.
\end{abstract}

Index Terms: Musa, Cultivar, Anthracnose, Postharvest Diseases.

\footnotetext{
(Trabalho 056-09). Recebido em :27-02-2009. Aceito para publicação em : 06-04-2010.

${ }^{2}$ Mestrando em Fitopatologia, Universidade Federal de Viçosa, Av PH Rolfs S/n, 36571-000

${ }^{3}$ Eng $^{\circ}$. Agrônomo, D.Sc. Professor da Universidade Estadual de Montes Claros, Câmpus de Janaúba, Av. Reinaldo Viana 2630, Bico da Pedra, 39440-000. Bolsista da Fapemig. edson.mizobutsi@unimontes.br

${ }^{4}$ Pesquisador da Embrapa Mandioca e Fruticultura Tropical, C.P.007, 44380-000, Cruz das Almas-BA. ssilva@cnpmf.embrapa.br ${ }^{5}$ Eng $^{\circ}$. Agrônomo, D.Sc. Professor da Universidade Estadual de Montes Claros, Câmpus de Janaúba, Av. Reinaldo Viana 2630, Bico da Pedra, 39440-000. sidnei.reis@unimontes.br

${ }^{6} E_{n g}$. Agrônomo, D.Sc. Professora da Universidade Estadual de Montes Claros, Câmpus de Janaúba, Av. Reinaldo Viana 2630, Bico da Pedra, 39440-000. gisele.mizobutsi@unimontes.br

${ }^{7}$ Eng $^{\circ}$. Agrônomo, D.Sc. Professora da Universidade Estadual de Montes Claros, Câmpus de Janaúba, Av. Reinaldo Viana 2630, Bico da Pedra, 39440-000. adelica@unimontes.br

${ }^{8}$ Eng $^{\circ}$. Agrônomo, D.Sc. Professora da Universidade Estadual de Montes Claros, Câmpus de Janaúba, Av. Reinaldo Viana 2630, Bico da Pedra, 39440-000. regina.ribeiro@unimontes.br

${ }^{9} \mathrm{Eng}^{\circ}$. Agrônomo, D.Sc. Professor da Universidade Estadual de Montes Claros, Câmpus de Janaúba, Av. Reinaldo Viana 2630, Bico da Pedra, 39440-000. Victor.maia@unimontes.br
} 


\section{INTRODUÇÃO}

O Brasil é o segundo maior produtor mundial de bananas, entretanto a participação brasileira no mercado externo é insignificante devido ao elevado consumo interno e perdas substanciais na fase de póscolheita (MAIA et al., 2008). As perdas devem-se principalmente ao baixo nível tecnológico adotado nos pomares (MAIA et al., 2008; SILVA et al., 2003; SOUZA; TORRES FILHO, 1999) e às doenças (PEREIRA et al., 1999). Destas, destacam-se as podridões em pós-colheita que podem ocasionar perdas de 40 a 60\% (LICHTEMBERG, 1999).

Dentre as podridões em pós-colheita, a antracnose causada por Colletotrichum musae (BERK; CURTIS) von Arx é a principal doença responsável pela deterioração da fruta durante o transporte, armazenamento e comercialização (MORAES et al., 2008; PESSOA et al., 2007; MORAES et al., 2006). A doença ocorre principalmente na fase de maturação, porém a infecção inicia-se no campo, ocasião em que os conídios do agente causal, dispersos no ar, infectam os frutos. Essa infecção permanece quiescente até o início da maturação. As duas formas distintas dessa doença são a antracnose latente originária da infecção quiescente e a antracnose não latente, produzida pela invasão do patógeno, principalmente por intermédio dos ferimentos ocasionados nos frutos verdes em trânsito (CORDEIRO et al., 2004).

A cultivar Prata-Anã é amplamente plantada no Brasil, apesar de não possuir resistência às principais doenças que afetam a planta (SILVA et al., 2004). Provavelmente, as cultivares tradicionais serão substituídas por causa da suscetibilidade a doenças como Sigatoka-Amarela, Sigatoka-Negra, Mal-do-Panamá e Moko (SILVA;ALVES, 1999).

Apesar de Snowdon (1990) relatar que alguns híbridos tetraploides apresentam resistência à antracnose, são reduzidas as informações sobre a resistência desses genótipos e o comportamento dos mesmos no Brasil. Entretanto, alguns trabalhos, como os de Pinho et al. (2007), relatam que a cultivar Pakovan Ken apresentou maior severidade da antracnose e a cultivar Thap Maeo apresentou menor severidade a essa doença.

A ausência de estudos sobre a epidemiologia de doenças em frutos de bananeiras tem dificultado o esclarecimento da influência da concentração de inóculo no progresso da podridão de antracnose dessa fruta. Segundo Chakrabotry, (1990), maiores concentrações de inóculo promovem aumento da penetração do fungo, resultando em maior severidade de doença. Desse modo, o presente trabalho teve como objetivo avaliar o comportamento de diferentes genótipos de bananeiras resistentes à Sigatoka-Negra em função de diferentes concentrações de inóculo de Colletotrichum musae aplicadas nos frutos quanto à incidência e severidade da antracnose.

\section{MATERIAL E MÉTODOS}

Os cachos de banana foram provenientes do município de Sebastião Laranjeira-BA. Foram colhidos em estádio pré-climatérico, despencados e selecionadas as pencas centrais dos cachos, visando à maior uniformidade dos frutos durante a maturação em pós-colheita. A seguir, os frutos foram transportados para o Laboratório de Patologia Pós-colheita da Unimontes - Câmpus de Janaúba-MG. Os genótipos utilizados no teste foram: Thap Maeo, Caipira, Pacovan Ken, Ambrosia, PV 42-53, PA 42-44, FHIA 01, FHIA 18 e a cultivar Prata-Anã utilizada como testemunha, que é suscetível à antracnose.

O isolado de Colletotrichum musae foi obtido de frutos de bananeira da cv. Prata-Anã, exibindo sintomas típicos da antracnose e isolados em meio batata-dextrose-ágar (BDA), incubado a 25 ${ }^{\circ} \mathrm{C}$. Foram preparadas suspensões de conídios pela adição de água destilada esterilizada à superfície das culturas, filtragem em camada dupla de gaze e ajuste da concentração em câmara de Neubauer para $10^{2} ; 10^{3} ; 10^{4} ; 10^{5}$ e $10^{6}$ conídios $/ \mathrm{mL}$. As inoculações foram realizadas em buquês contendo três frutos, com diferentes concentrações de conídios de C. musae e mantidos em câmara a $25^{\circ} \mathrm{C}$. A evolução da cor dos buquês foi determinada com auxílio da escala visual descrita por Soto Ballestero (1992). A incidência foi determinada pela ausência ou presença de sintomas de antracnose aos 3; 6; 9 e 12 dias após a inoculação (D.A.I.) dos frutos. A severidade da doença também foi avaliada aos 3; 6;9 e 12 DAI com o auxílio de uma escala diagramática descrita por Moraes et al. (2008).

$\mathrm{O}$ delineamento experimental utilizado foi o inteiramente casualizado, em esquema fatorial 9x6 64 (cultivares $\mathrm{x}$ concentrações de esporos $\mathrm{x}$ épocas de avaliação), com quatro repetições contendo cada uma quatro buquês de três frutos. Devido às variáveis serem discretas, testaram-se as hipóteses básicas para a realização da análise de variância, aditividade, normalidade do erro e homogeneidade da variância. Uma vez não atendendo a estes princípios, adotou-se a estatística não paramétrica, utilizando-se do teste de Kruskal-Wallis, por meio do procedimento NPAR1WAY do software SAS (SAS INSTITUTE, 2000). Quando significativo, as médias dos tratamentos para cada variável foram comparadas pelo teste de "P" do 
Contraste, por meio do procedimento MULTTEST do software SAS (SAS INSTITUTE, 2000).

\section{RESULTADOS E DISCUSSÃO}

As cultivares e híbridos avaliados não apresentaram resistência à antracnose, variando em níveis de incidência e severidades.

Aos três e seis DAI, não houve incidência e severidade da antracnose nos frutos em função do dia de avaliação dentro de cada concentração de inóculo e genótipo. Isso se deve ao fato de a infecção permanecer quiescente até o início da maturação dos frutos, ocasião em que os sintomas começam a desenvolverse (CORDEIRO et al., 2004). As cultivares Caipira e Thap Maeo não inoculadas com $C$. musae não apresentaram diferenças na incidência de antracnose entre os seis e nove DAI. Ao avaliar os frutos, aos nove e doze DAI, pôde-se observar a incidência de antracnose em todos os frutos das cultivares PrataAnã, Ambrosia, FHIA 01 e FHIA 18.

Os valores da incidência de antracnose em função da concentração de conídios para as cultivares Prata-Anã, Pacovan Ken, Ambrosia, FHIA 01, FHIA 18 e híbridos PV 42-53, PA 42-44, em todos os dias avaliados, não foram significativos $(\mathrm{P}<0,05)$. De acordo a Tabela 1, as cultivares Caipira e Thap Maeo apresentaram diferenças entre as concentrações de conídios, aos nove e doze DAI, onde se observou menor incidência de antracnose, para o tratamento onde não houve inoculação com $C$. musae.

Ao se compararem os genótipos dentro de cada concentração de esporos e dia de avaliação, pode-se observar pela Tabela 1, que aos nove DAI, a cultivar Caipira apresentou menor incidência de antracnose quando se utilizaram $10^{2} ; 10^{3}$ e $10^{5}$ conídios/ $\mathrm{mL}$, enquanto o híbrido PA 42-44 apresentou menor incidência de $C$. musae para todos os tratamentos onde se utilizaram diferentes concentrações de conídios $(\mathrm{P}<0,05)$. Porém, para os frutos onde não houve inoculação com C. musae, as diferenças na incidência de antracnose ocorreram aos doze DAI, quando as cultivares Caipira e Thap Maeo apresentaram a menor incidência de antracnose.

A severidade da antracnose em função do dia de avaliação dentro de cada concentração de conídios e genótipo apresentou diferenças entre as avaliações realizadas aos nove e doze DAI. Também se observou significância $(\mathrm{P}<0,05)$ quando se avaliaram as concentrações de conídios dentro de cada variedade e dia de avaliação. Aos doze DAI, o tratamento-testemunha foi o que apresentou menor severidade de antracnose para todos os genótipos estudados (Tabela 1). Esses resultados evidenciam a ocorrência da infecção por
C. musae no campo de produção, indicando que os frutos já estavam infectados. Frutos inoculados com $10^{6}$ conídios $/ \mathrm{mL}$ de $C$. musae apresentaram maior severidade da antracnose nas cultivares Pacovan Ken, Ambrosia, FHIA 01, FHIA 18 e Thap Maeo, enquanto na cultivar Caipira e no híbrido PA 42-44, os tratamentos inoculados com $10^{5}$ e $10^{6}$ conídios/ $\mathrm{mL}$ não proporcionaram diferenças estatísticas entre a severidade de antracnose. Não houve diferenças significativas na severidade de antracnose em frutos da cultivar Prata-Anã e no híbrido PV 42-53 inoculados com $10^{4}$ conídios $/ \mathrm{mL}$ de C. musae.

Houve interação significativa $(\mathrm{P}<0,05)$ de genótipos, concentração de conídios e tempo de avaliação após a inoculação. A cultivar Thap Maeo apresentou menor severidade de antracnose para os frutos inoculados com $0 ; 10^{3} ; 10^{4} ; 10^{5}$ e $10^{6}$ conídios/ $\mathrm{mL}$ de $C$. musae, avaliados aos $12 \mathrm{DAI}$, variando de $1,42 \%$ a $27,50 \%$ da área lesionada do fruto para os tratamentos com 0 e $10^{6}$ conídios $/ \mathrm{mL}$ de $C$. musae, respectivamente (Tabela 1). Quando se inocularam os frutos com $10^{2}$ conídios $/ \mathrm{mL}$, a cultivar Caipira e o híbrido PA 42-44 apresentaram menor severidade de antracnose. A cultivar Caipira apresentou menor severidade de antracnose quando se utilizaram até $10^{3}$ conídios $/ \mathrm{mL}$ de $C$. musae, porém, a partir dessa concentração de inóculo, os frutos tornaram-se muito suscetíveis à doença, chegando a apresentar $41 \%$ da área lesionada dos frutos. As cultivares mais suscetíveis à antracnose foram a Ambrosia e a FHIA 18 com $64 \%$ e $64 \%$ e o híbrido PV $42-53$ com $61,33 \%$ da área do fruto lesionada, respectivamente. 
TABELA 1 - Percentagem de incidência e severidade de antracnose em função da presença ou ausência de sintomas nos frutos de genótipos de bananeira submetidos à inoculação com diferentes concentrações de inóculo de Colletotrichum musae, em diferentes dias de avaliação.

\begin{tabular}{|c|c|c|c|c|c|}
\hline \multirow[b]{3}{*}{ Genótipos } & \multirow{3}{*}{$\begin{array}{c}\text { Inóculo } \\
\text { (conídios/mL) }\end{array}$} & \multicolumn{4}{|c|}{ Dias após a inoculação dos frutos } \\
\hline & & \multicolumn{2}{|c|}{ Incidência } & \multicolumn{2}{|c|}{ Severidade } \\
\hline & & 9 & 12 & 9 & 12 \\
\hline \multirow[t]{6}{*}{ Prata-Anã } & 0 & $100 \mathrm{Aa}^{1}$ & $100 \mathrm{Aa}$ & $2,35 \mathrm{Aa}$ & $6,92 \mathrm{Ba}$ \\
\hline & $10^{2}$ & $100 \mathrm{Aa}$ & $100 \mathrm{Aa}$ & $4,86 \mathrm{Aa}$ & $26,50 \mathrm{Bb}$ \\
\hline & $10^{3}$ & $100 \mathrm{Aa}$ & $100 \mathrm{Aa}$ & $9,08 \mathrm{Ab}$ & $35,33 \mathrm{Bb}$ \\
\hline & $10^{4}$ & $100 \mathrm{Aa}$ & $100 \mathrm{Aa}$ & $10,57 \mathrm{Ab}$ & $46,67 \mathrm{Bc}$ \\
\hline & $10^{5}$ & $100 \mathrm{Aa}$ & $100 \mathrm{Aa}$ & $12,71 \mathrm{Abc}$ & $52,00 \mathrm{Bc}$ \\
\hline & $10^{6}$ & $100 \mathrm{Aa}$ & $100 \mathrm{Aa}$ & $14,96 \mathrm{Ac}$ & $54,67 \mathrm{Bc}$ \\
\hline \multirow[t]{6}{*}{ Caipira } & 0 & $0 \mathrm{Aa}$ & $56 \mathrm{Ba}$ & $0,00 \mathrm{Aa}$ & $1,50 \mathrm{Ba}$ \\
\hline & $10^{2}$ & $35 \mathrm{Ab}$ & $100 \mathrm{Bb}$ & $0,90 \mathrm{Aab}$ & $11,04 \mathrm{Bb}$ \\
\hline & $10^{3}$ & $44 \mathrm{Ab}$ & $100 \mathrm{Bb}$ & $1,21 \mathrm{Aab}$ & $14,54 \mathrm{Bb}$ \\
\hline & $10^{4}$ & $92 \mathrm{Ac}$ & $100 \mathrm{Bb}$ & $2,60 \mathrm{Abc}$ & $31,33 \mathrm{Bc}$ \\
\hline & $10^{5}$ & $85 \mathrm{Ac}$ & $100 \mathrm{Bb}$ & $4,31 \mathrm{Ac}$ & $37,33 \mathrm{Bd}$ \\
\hline & $10^{6}$ & $96 \mathrm{Ac}$ & $100 \mathrm{Ab}$ & $6,54 \mathrm{Ad}$ & $41,00 \mathrm{Bd}$ \\
\hline \multirow[t]{6}{*}{ Pacovan Ken } & 0 & $98 \mathrm{Aa}$ & $100 \mathrm{Aa}$ & $3,47 \mathrm{Aa}$ & $12,67 \mathrm{Ba}$ \\
\hline & $10^{2}$ & $100 \mathrm{Aa}$ & $100 \mathrm{Aa}$ & $7,39 \mathrm{Aab}$ & $28,67 \mathrm{Bb}$ \\
\hline & $10^{3}$ & $100 \mathrm{Aa}$ & $100 \mathrm{Aa}$ & 7,73 Aab & $29,67 \mathrm{Bb}$ \\
\hline & $10^{4}$ & $100 \mathrm{Aa}$ & $100 \mathrm{Aa}$ & 7,76 Aab & $30,00 \mathrm{Bb}$ \\
\hline & $10^{5}$ & $94 \mathrm{Aa}$ & $100 \mathrm{Ba}$ & $9,41 \mathrm{Abc}$ & $32,50 \mathrm{Bb}$ \\
\hline & $10^{6}$ & $100 \mathrm{Aa}$ & $100 \mathrm{Aa}$ & $13,58 \mathrm{Ac}$ & $46,33 \mathrm{Bc}$ \\
\hline \multirow[t]{6}{*}{ Thap Maeo } & 0 & $15 \mathrm{Aa}$ & $56 \mathrm{Ba}$ & $0,09 \mathrm{Aa}$ & $1,42 \mathrm{Ba}$ \\
\hline & $10^{2}$ & $94 \mathrm{Ab}$ & $100 \mathrm{Bb}$ & $4,25 \mathrm{Ab}$ & $16,21 \mathrm{Bb}$ \\
\hline & $10^{3}$ & $96 \mathrm{Ab}$ & $100 \mathrm{Ab}$ & $5,73 \mathrm{Abc}$ & $19,67 \mathrm{Bb}$ \\
\hline & $10^{4}$ & $94 \mathrm{Ab}$ & $100 \mathrm{Bb}$ & $6,31 \mathrm{Abc}$ & $21,17 \mathrm{Bb}$ \\
\hline & $10^{5}$ & $94 \mathrm{Ab}$ & $100 \mathrm{Bb}$ & 7,08 Ac & $21,83 \mathrm{Bbc}$ \\
\hline & $10^{6}$ & $92 \mathrm{Ab}$ & $100 \mathrm{Bb}$ & 7,94 Ac & $27,50 \mathrm{Bc}$ \\
\hline \multirow[t]{6}{*}{ PV 42-53 } & 0 & $83 \mathrm{Aa}$ & $100 \mathrm{Aa}$ & $3,58 \mathrm{Aa}$ & $11,67 \mathrm{Ba}$ \\
\hline & $10^{2}$ & $100 \mathrm{Aa}$ & $100 \mathrm{Aa}$ & $6,00 \mathrm{Aa}$ & $33,33 \mathrm{Bb}$ \\
\hline & $10^{3}$ & $100 \mathrm{Aa}$ & $100 \mathrm{Aa}$ & $10,50 \mathrm{Aab}$ & $40,00 \mathrm{Bb}$ \\
\hline & $10^{4}$ & $100 \mathrm{Aa}$ & $100 \mathrm{Aa}$ & $18,00 \mathrm{Abc}$ & $53,33 \mathrm{Bc}$ \\
\hline & $10^{5}$ & $100 \mathrm{Aa}$ & $100 \mathrm{Aa}$ & $25,33 \mathrm{Ac}$ & $56,00 \mathrm{Bc}$ \\
\hline & $10^{6}$ & $100 \mathrm{Aa}$ & $100 \mathrm{Aa}$ & $49,33 \mathrm{Ad}$ & $64,00 \mathrm{Bc}$ \\
\hline \multirow[t]{6}{*}{ PA 42-44 } & 0 & $50 \mathrm{Aa}$ & $100 \mathrm{Ba}$ & $1,00 \mathrm{Aa}$ & $4,67 \mathrm{Ba}$ \\
\hline & $10^{2}$ & $44 \mathrm{Aa}$ & $100 \mathrm{Ba}$ & $1,33 \mathrm{Aa}$ & $12,17 \mathrm{Bb}$ \\
\hline & $10^{3}$ & $39 \mathrm{Aa}$ & $100 \mathrm{Ba}$ & $1,39 \mathrm{Aa}$ & $18,56 \mathrm{Bbc}$ \\
\hline & $10^{4}$ & $67 \mathrm{Aa}$ & $100 \mathrm{Ba}$ & $2,61 \mathrm{Aab}$ & $24,44 \mathrm{Bc}$ \\
\hline & $10^{5}$ & $75 \mathrm{Aa}$ & $100 \mathrm{Ba}$ & $4,17 \mathrm{Abc}$ & $44,44 \mathrm{Bd}$ \\
\hline & $10^{6}$ & $83 \mathrm{Aa}$ & $100 \mathrm{Ba}$ & $5,89 \mathrm{Ac}$ & $46,67 \mathrm{Bd}$ \\
\hline \multirow[t]{6}{*}{ Ambrosia } & 0 & $100 \mathrm{Aa}$ & $100 \mathrm{Aa}$ & $3,52 \mathrm{Aa}$ & $11,00 \mathrm{Ba}$ \\
\hline & $10^{2}$ & $100 \mathrm{Aa}$ & $100 \mathrm{Aa}$ & $7,42 \mathrm{Aa}$ & $31,00 \mathrm{Bb}$ \\
\hline & $10^{3}$ & $100 \mathrm{Aa}$ & $100 \mathrm{Aa}$ & $9,50 \mathrm{Aa}$ & $35,33 \mathrm{Bb}$ \\
\hline & $10^{4}$ & $100 \mathrm{Aa}$ & $100 \mathrm{Aa}$ & $20,83 \mathrm{Ab}$ & $48,00 \mathrm{Bc}$ \\
\hline & $10^{5}$ & $100 \mathrm{Aa}$ & $100 \mathrm{Aa}$ & $34,00 \mathrm{Ac}$ & $52,00 \mathrm{Bc}$ \\
\hline & $10^{6}$ & $100 \mathrm{Aa}$ & $100 \mathrm{Aa}$ & $52,67 \mathrm{Ad}$ & $64,00 \mathrm{Bd}$ \\
\hline \multirow[t]{6}{*}{ FHIA 01} & 0 & $100 \mathrm{Aa}$ & $100 \mathrm{Aa}$ & $2,00 \mathrm{Aa}$ & $5,83 \mathrm{Ba}$ \\
\hline & $10^{2}$ & $100 \mathrm{Aa}$ & $100 \mathrm{Aa}$ & $5,33 \mathrm{Aa}$ & $22,08 \mathrm{Bb}$ \\
\hline & $10^{3}$ & $100 \mathrm{Aa}$ & $100 \mathrm{Aa}$ & $5,75 \mathrm{Aa}$ & $34,67 \mathrm{Bc}$ \\
\hline & $10^{4}$ & $100 \mathrm{Aa}$ & $100 \mathrm{Aa}$ & $13,83 \mathrm{Ab}$ & $50,00 \mathrm{Bd}$ \\
\hline & $10^{5}$ & $100 \mathrm{Aa}$ & $100 \mathrm{~A}$ & $24,50 \mathrm{Ac}$ & $52,00 \mathrm{Bd}$ \\
\hline & $10^{6}$ & $100 \mathrm{Aa}$ & $100 \mathrm{Aa}$ & $29,67 \mathrm{Ad}$ & $58,00 \mathrm{Be}$ \\
\hline \multirow[t]{6}{*}{ FHIA 18} & 0 & $100 \mathrm{Aa}$ & $100 \mathrm{Aa}$ & $3,04 \mathrm{Aa}$ & $6,60 \mathrm{Ba}$ \\
\hline & $10^{2}$ & $100 \mathrm{Aa}$ & $100 \mathrm{Aa}$ & $6,25 \mathrm{Aab}$ & $27,83 \mathrm{Bb}$ \\
\hline & $10^{3}$ & $100 \mathrm{Aa}$ & $100 \mathrm{Aa}$ & $9,25 \mathrm{Ab}$ & $47,67 \mathrm{Bc}$ \\
\hline & $10^{4}$ & $100 \mathrm{Aa}$ & $100 \mathrm{Aa}$ & $17,83 \mathrm{Ac}$ & $51,33 \mathrm{Bcd}$ \\
\hline & $10^{5}$ & $100 \mathrm{Aa}$ & $100 \mathrm{Aa}$ & $30,00 \mathrm{Ad}$ & $56,00 \mathrm{Bde}$ \\
\hline & $10^{6}$ & $100 \mathrm{Aa}$ & $100 \mathrm{Aa}$ & $44,33 \mathrm{Ae}$ & $61,33 \mathrm{Be}$ \\
\hline
\end{tabular}

${ }^{1}$ Médias seguidas de mesma letra maiúscula na linha e minúscula na coluna dentro de cada genótipo não diferem entre si, pelo teste de "P" do Contraste, ao nível de significância de 5\% de probabilidade. 


\section{CONCLUSÕES}

1-Todas as cultivares analisadas são suscetíveis à antracnose, nas diferentes concentrações de inóculo.

2-As cultivares Caipira e Thap Maeo apresentam menor incidência de antracnose quiescente nos frutos não inoculados.

3-As cultivares Ambrosia e FHIA 18 e o híbrido PV 42-53 apresentam a maior severidade da doença com $64 \%, 61,33 \%$ e $64 \%$, respectivamente.

\section{AGRADECIMENTO}

Os autores agradecem à Fundação de Amparo à Pesquisa do Estado de Minas Gerais - FAPEMIG, pelo apoio indispensável para a realização do trabalho.

\section{REFERÊNCIAS}

CHAKRABORTY, S. Expression of quantitative resistance to Colletotrichum gloeosporioides in Stylosanthes scabra at different inoculum concentrations and day night temperatures. Australasian Journal of Agricultural Research, Collingwood, v.41, p.89-100, 1990.

CORDEIRO, Z.J.M.; MATOS, A.P.de; MEISSNER FILHO, P.E. Doenças e métodos de controle. In: BORGES, A.L.; SOUZA, L. da S. O cultivo da bananeira. Cruz das Almas: Embrapa-CNPMF, 2004. p.146-182.

LICHTEMBERG, L.A. Colheita e pós-colheita da banana. Informe Agropecuário, Belo Horizonte, v.20, n. 196, p.73-90, 1999.

MAIA, V.M.; SALOMÃO, L.C.C.; SIQUEIRA, D.L.; PUSCHMANN, R.; MOTA FILHO, V.J.G. CECON, P.R. Tipos e intensidade de danos mecânicos em bananas 'prata-anã' ao longo da cadeia de comercialização. Revista Brasileira de Fruticultura, Jaboticabal, v. 30, n. 2, p. 365-370, 2008.

MORAES, W. S.; ZAMBOLIM, L.; LIMA, J. D. Incidência de fungos em pós-colheira de banana (Musa spp.) 'Prata-anã' (AAB). Summa Phytopathologica, Botucatu, v. 32, n. 1, p. 67-70, 2006.

MORAES, W. S.; ZAMBOLIM, L. ; LIMA, J.D. Quimioterapia de banana "Prata-anã" no controle de podridão pós-colheita. Arquivos Instituto Biológico, São Paulo, v.75, n.1, p.79-84, 2008.
PEREIRA, L.V.; CORDEIRO, Z.J.M.; FIGUEIRA, A. dos R.; HINZ, R.H.; MATOS, A.P. Doenças da bananeira. Informe Agropecuário, Belo Horizonte, v.20, n.196, p.37-47, 1999.

PESSOA, W.R.L.S; OLIVEIRA, S.M.A.; DANTAS, S.A.F.; TAVARES, S.C.C. DE H.; SANTOS, A.M.G. Efeito da temperatura e período de molhamento sobre o desenvolvimento de lesões de Colletotrichum musae em banana. Summa Phytopathologica, Botucatu, v.33, n.2, p.147-151, 2007.

PINHO, D.B.; MIZOBUTSI, E.H.; LEMOS, J.P.; REIS, S.T.; REIS, R.V.; OLIVEIRA, D.; POLETE-MIZOBUTSI, G.; XAVIER, A.A.; RIBEIRO, R.C.F. Suscetibilidade à antracnose de três cultivares de bananeiras resistentes a SigatokaNegra. In: CONGRESSO BRASILEIRO DE FITOPATOLOGIA, 32., 2007, Maringá. Anais.. Maringá: UEM, 2007. p.191.

SAS INSTITUTE. SAS/STAT User's guide. Version 8. Cary, 2000.

SILVA, C.S.; PEROSA, J.M.Y.; RUA, P.C.; ABREU, C.L.M.; PANTANO, S.C.; VIEIRA, C.R.Y.I.; BRIZOLA, R.M.O. Avaliação econômica das perdas de banana no mercado varejista: um estudo de caso. Revista Brasileira de Fruticultura, Jaboticabal, v.25, n.2, p.228-234, 2003.

SILVA, S.de O. e; ALVES, E.J. Melhoramento genético e novas cultivares de bananeira. Informe Agropecuário, Belo Horizonte, v.20, n.196, p.9196, 1999.

SILVA, S. de O.e; SANTOS-SEREJO, J.A. dos; CORDEIRO, Z.J.M. Variedades. In: BORGES, A.L.; SOUZA, L. da S. O cultivo da bananeira. Cruz das Almas: Embrapa-CNPMF, 2004. p. 45-58.

SNOWDON, A.L. A color Atlas of postharvest disease $\&$ disoders of fruits $\&$ vegetable. London: CRC Press, 1990. v1, 302 p.

SOTO BALLESTERO, M. Bananos: Cultivo y comercializacion. San Jose: Litografia e Imprenta Lil, 1992. 674p.

SOUZA, J.S.; TORRES FILHO, P. Aspectos Socioeconômicos. In: ALVES, E. J. A cultura da banana: aspectos técnicos, socioeconômicos e agroindustriais. 2. ed., Cruz das Almas: Embrapa-CNPMF, 1999. p.507-524. 